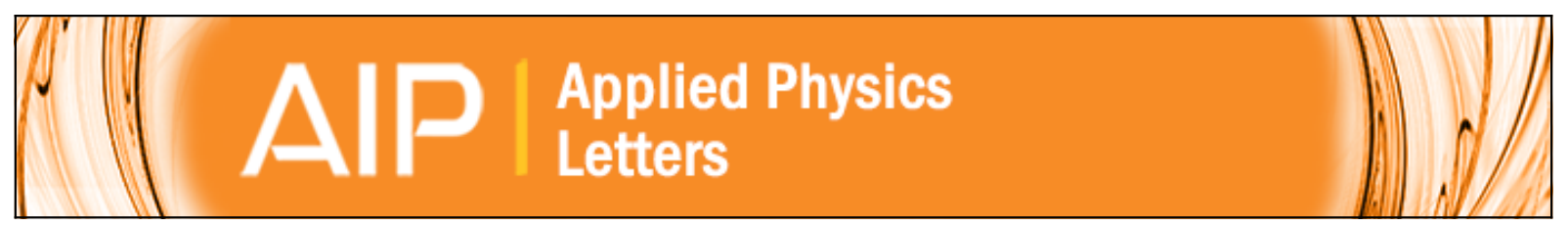

\title{
Effects of surface oxide formation on germanium nanowire band-edge photoluminescence
}

Fatemeh Sadat Minaye Hashemi, Shruti Thombare, Anna Fontcuberta i Morral, Mark L. Brongersma, and Paul

C. McIntyre

Citation: Applied Physics Letters 102, 251122 (2013); doi: 10.1063/1.4812334

View online: http://dx.doi.org/10.1063/1.4812334

View Table of Contents: http://scitation.aip.org/content/aip/journal/apl/102/25?ver=pdfcov

Published by the AIP Publishing

\section{Articles you may be interested in}

Microstructural characteristics and phonon structures in luminescence from surface oxidized Ge nanocrystals embedded in $\mathrm{HfO} 2$ matrix

J. Appl. Phys. 108, 053510 (2010); 10.1063/1.3475717

Photoemission studies of passivation of germanium nanowires

Appl. Phys. Lett. 87, 263109 (2005); 10.1063/1.2158027

Formation mechanism and optical properties of nanocrystalline silicon in silicon oxide J. Appl. Phys. 98, 014303 (2005); 10.1063/1.1935136

Alternative surface passivation on germanium for metal-oxide-semiconductor applications with high- $\mathrm{k}$ gate dielectric

Appl. Phys. Lett. 85, 4127 (2004); 10.1063/1.1812835

Fabrication and photoluminescence investigation of silicon nanowires on silicon-on-insulator material

J. Vac. Sci. Technol. B 16, 582 (1998); 10.1116/1.589867

\section{AlP Re-register for Table of Content Alerts}




\title{
Effects of surface oxide formation on germanium nanowire band-edge photoluminescence
}

\author{
Fatemeh Sadat Minaye Hashemi, ${ }^{1,2}$ Shruti Thombare, ${ }^{1}$ Anna Fontcuberta i Morral, ${ }^{2}$ \\ Mark L. Brongersma, ${ }^{1}$ and Paul C. Mclntyre ${ }^{1,3}$ \\ ${ }^{1}$ Department of Materials Science and Engineering, Stanford University, Stanford, California 94305, USA \\ ${ }^{2}$ Laboratoire des Matériaux Semiconducteurs, Ecole Polytechnique Fédérale de Lausanne, 1015 Lausanne, \\ Switzerland \\ ${ }^{3}$ Geballe Laboratory for Advanced Materials, Stanford University, Stanford, California 94305, USA
}

(Received 8 April 2013; accepted 5 June 2013; published online 28 June 2013)

\begin{abstract}
The effect of intentional surface oxide formation on band-edge photoluminescence (PL) of Ge nanowires was investigated. Thermal oxidation in molecular $\mathrm{O}_{2}$ was used to produce a surface oxide layer on assemblies of single crystal nanowires grown by the vapor-liquid-solid method. With increasing oxidation of the wires, the band-edge PL associated with the indirect gap transition becomes more intense. X-ray photoelectron spectroscopy confirms the formation of an increasingly $\mathrm{GeO}_{2}$-like surface oxide under annealing conditions that enhance the indirect-gap PL, consistent with surface oxide passivation of nonradiative recombination centers initially present on the nanowire surface. @ 2013 AIP Publishing LLC. [http://dx.doi.org/10.1063/1.4812334]
\end{abstract}

Nanoscale semiconductor structures such as quantum wells and nanowires (NWs) have been used and studied intensively for electronic and optoelectronic applications. ${ }^{1}$ Germanium (Ge) nanostructures are of particular interest due to their high carrier mobilities, compatibility with silicon-based integrated circuits, and the favorable bulk Ge bandgap for photonic applications. This latter property provides motivation to investigate such nanostructures as building blocks for light sources ${ }^{2}$ and detectors ${ }^{3,4}$ operating at standard telecommunication wavelengths. Surface and interface defects on large surface area-to-volume ratio Ge nanostructures can trap and scatter carriers, limiting the performance of such nanostructures in both electronic and photonic applications. This prompts interest in methods to study and passivate such defects, the subject of the experiments reported herein.

Photoluminescence (PL) measurements of semiconductor nanostructures can provide valuable information about their electronic structure and interface characteristics. ${ }^{5}$ Several groups have reported the observation of near-infrared (NIR) PL from Ge quantum wells. ${ }^{6-10}$ Other studies have mainly concerned visible PL from Ge nanocrystals embedded in various oxide matrices, and the role of the defects at the nanocrystal/ matrix interface or in the matrix itself. ${ }^{11-16}$

In the case of Ge NWs, there are few reports of PL detected for band-to-band transitions in such nanowires, ${ }^{17}$ due to the difficulty of detecting emission from unpassivated $\mathrm{Ge}$ NWs, which results from carrier trapping and nonradiative recombination. Among these, coherently strained Ge core/ $\mathrm{SiGe}$ shell nanowires exhibiting strong and bulk-like bandedge PL have been reported recently. ${ }^{18}$ Also, we have recently observed near-infrared PL from freestanding Ge nanowires originating from direct-bandgap recombination..$^{19}$ Because the direct bandgap radiative recombination rate is much greater than indirect one, photogenerated electrons can recombine radiatively via the direct bandgap and PL signal can be detected via this process even for Ge NWs with unpassivated surfaces. However, nonradiative recombination via surface defects very effectively quenches PL from Ge NWs via the slow, phonon-mediated indirect gap transition. ${ }^{19}$

Germanium forms a native oxide of somewhat ill-defined composition when exposed to the air. ${ }^{20} \mathrm{~A}$ defective $\mathrm{GeO}_{\mathrm{x}}$ (suboxide)/Ge interface has "fast" interface states (ps-ns response time) that will tend to quench radiative recombination of photogenerated carrers. ${ }^{21-23}$ In contrast, a $\mathrm{GeO}_{2} / \mathrm{Ge}$ interface provides a lower interface state density than a Ge suboxide/Ge interface. ${ }^{21-23}$ Obtaining higher oxidation states (e.g., $\mathrm{Ge}^{4+}$ ) in the oxide layer requires breaking additional Ge-Ge bonds, the rate of which can be accelerated by thermal annealing. ${ }^{20,24,25}$ Here, we have used annealing in $\mathrm{O}_{2}$ at moderate temperatures to control the state of oxidation of the Ge NW surface and to correlate the surface oxide stoichiometry with defect passivation using PL.

The Ge NWs were grown via a two-step growth process previously described in Ref. 26. Gold nanoparticles with $40 \mathrm{~nm}$ diameter were drop cast from colloidal solution on $\mathrm{Si}(111)$ (n-type dopant $\mathrm{P}$, resistivity ranging from 1500 to $2500 \Omega \cdot \mathrm{cm}$ ) substrates. The samples were heated to $375^{\circ} \mathrm{C}$ for $2 \mathrm{~min}$ for growth initiation and the temperature was lowered to $345^{\circ} \mathrm{C}$ for $20 \mathrm{~min}$. $\mathrm{GeH}_{4}$ diluted in $\mathrm{H}_{2}$ was used as a precursor with a total pressure of 30 Torr and a $\mathrm{GeH}_{4}$ partial pressure of 0.75 Torr.

Scanning electron microscopy (SEM) was used to characterize the growth direction, diameter, and length of the Ge NWs. The NWs are epitaxial with the substrate and follow a $\langle 111\rangle$ growth orientation. The Au catalyst particles were left on the tips of the Ge NWs after VLS growth. Selective Au etching ${ }^{27}$ after NW growth was not used, to avoid possibly altering the sidewall surface characteristics of the wires. As previously reported, ${ }^{28}$ the majority carrier (hole) concentration in these undoped Ge NWs is estimated to be about $10^{14} \mathrm{~cm}^{-3}$. The nanowires are likely p-type as a result of the acceptor character of Ge surface and interface states. SEM images such as the one in Fig. 1 show that most of the Ge NWs are vertically aligned on the $\mathrm{Si}$ (111) substrate with an average diameter of $\sim 40 \mathrm{~nm}$ and an average length of 


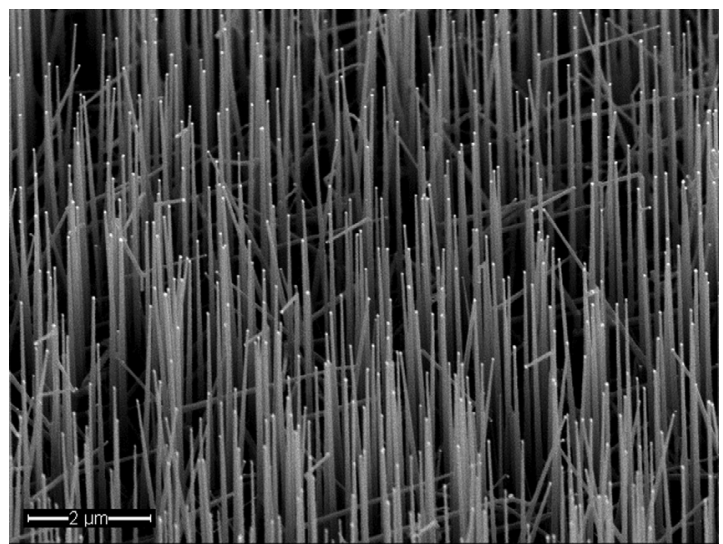

FIG. 1. SEM image of Au-catalyzed Ge NWs grown on a Si (111) substrate. Analysis of this image gives an average diameter and length of the Ge NWs of $40 \mathrm{~nm}$ and $6.5 \mu \mathrm{m}$, respectively.

$\sim 6.5 \mu \mathrm{m}$. Quantum confinement effects are expected to be negligible in these NWs because their diameter is larger than the exciton Bohr radius ( $\sim 24 \mathrm{~nm})$ of Ge. ${ }^{12}$

The PL setup consisted of a frequency-doubled Nd:YAG diode-pumped solid-state laser, emitting continuous wave at a wavelength of $532 \mathrm{~nm}$ (Spectra Physics Millennia Pro Laser) to excite the samples. The laser beam was focused onto the sample surfaces through a $10 \times$ microscope objective having a 0.26 numerical aperture. The incident laser power on the sample surfaces was varied between 10 and $40 \mathrm{~mW}$, resulting in laser power density between $\sim 3$ to $\sim 13 \mathrm{~W} / \mathrm{cm}^{2}$. The emitted light was dispersed in a Spectra Pro 2750 spectrometer in the wavelength range from 1100 to $2200 \mathrm{~nm}$ and collected by a liquid nitrogen-cooled strained InGaAs detector.

Fast trap states associated with defects at the nonstoichiometric $\mathrm{Ge}$ oxide/Ge interface ${ }^{29,30}$ will result in nonradiative recombination of photoexcited carriers. These trap levels are reported to have an energy level $\sim 0.15 \mathrm{eV}$ below the middle of the Ge bandgap. ${ }^{31}$ Due to the existence of these trap levels, the majority of photoexcited electrons in the L-valley of Ge NWs undergo non-radiative recombination and no PL signal can be detected from them. Formation of $\mathrm{GeO}_{2}$ bonding in the surface oxide may passivate ${ }^{21-23}$ these defects, removing trap levels from the bandgap of Ge. This permits photoluminescence of excited carriers by both direct and indirect bandgap transitions. Because Ge is an indirect bandgap material and the majority of electrons are excited into the L-valley, if these carriers recombine radiatively, the detection of a relatively intense indirect bandgap PL signal (at $1.77 \mu \mathrm{m}$ ) is expected. In the case of bulk Ge, the surface area-to-volume ratio is small and trap-related surface effects are negligible. Therefore, the PL signal from both indirect and direct bandgap carrier transitions can be detected in bulk Ge. ${ }^{19,32}$

Figure 2 shows a comparison between PL spectra detected from bulk Ge and Ge NWs with a native oxide formed by intentional exposure to lab air over a period of many days. Direct and indirect bandgap recombination peaks have been fitted with a Gaussian function ${ }^{33-35}$ to show the contribution of both transitions in the PL spectrum. The cumulative fit peak of two fitted direct and indirect bandgap peaks and experimental PL data have been intentionally offset along the vertical axis to make them more distinguishable.
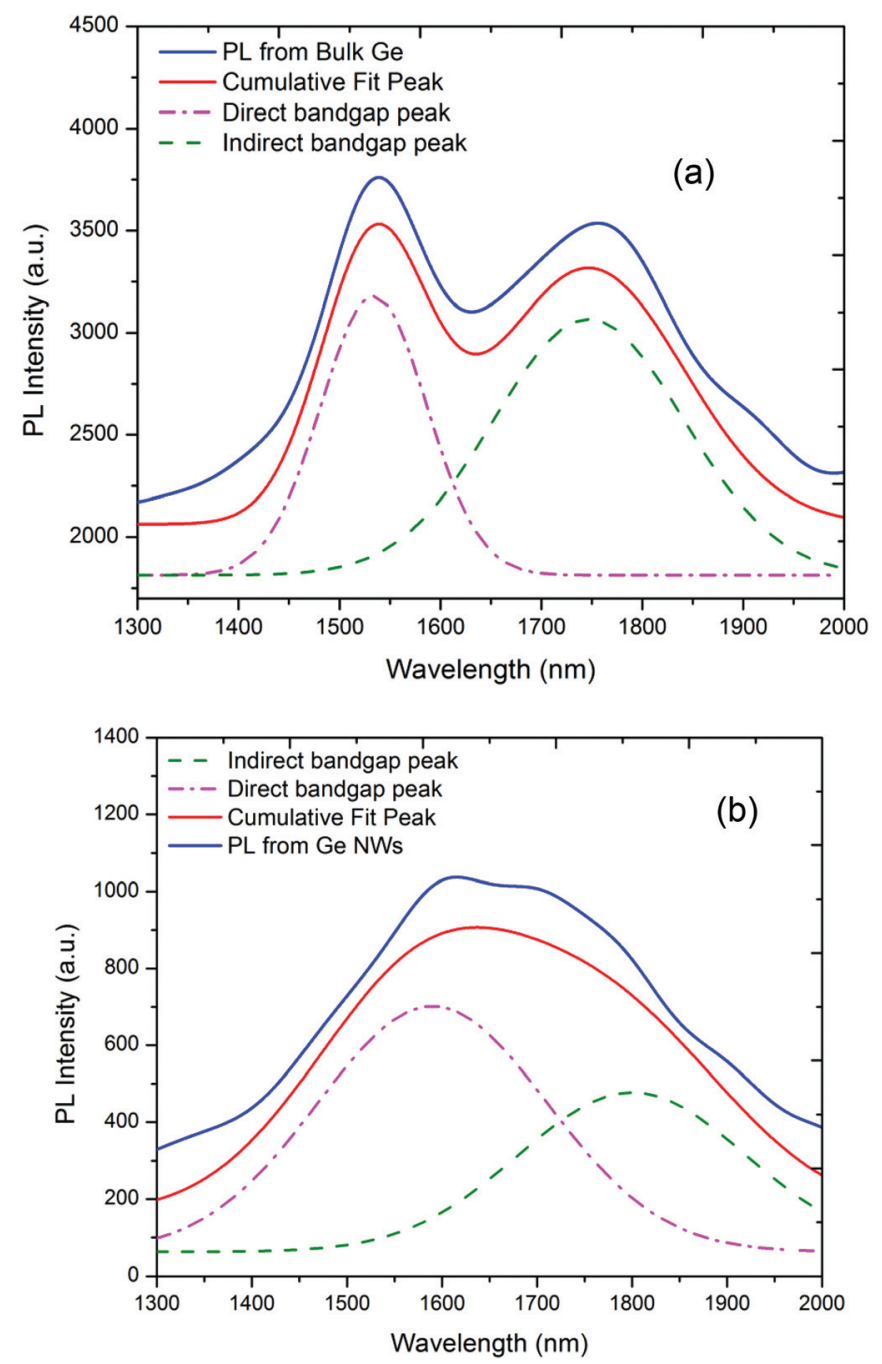

FIG. 2. NIR room-temperature PL signal from (a) bulk Ge and (b) Ge NWs with native oxide. (The fitting and experimental data curves have been intentionally offset along the vertical axis to make them distinguishable.)

It can be observed in Figure 2 that, for the Ge NWs with native oxide, radiative recombination via both the direct and indirect gap transitions contribute substantially, although the contribution of L-valley electrons to the PL signal is somewhat less than for the bulk sample. Also, in the case of Ge NWs, laser-induced heating produces a slight red shift for both direct and indirect bandgap recombination PL features. ${ }^{36-38}$ Both of the PL signals have been collected under the same conditions, with $20 \mathrm{~mW}$ laser power.

We annealed as-deposited Ge nanowire samples similar to the one in Figure 1 for different periods of time $(30 \mathrm{~min}$, $1 \mathrm{~h}, 2 \mathrm{~h}$, and $3 \mathrm{~h}$ ) in oxygen at atmospheric pressure at $220^{\circ} \mathrm{C}$. A practical maximum annealing temperature for such experiments is $361{ }^{\circ} \mathrm{C}$, the Au-Ge binary eutectic. Oxidation annealing at temperatures much above the eutectic will result in one-dimensional vapor-liquid solid oxidation of the $\mathrm{Ge}$ NWs to produce $\mathrm{GeO}_{\mathrm{x}} \mathrm{NWs}^{39}{ }^{39}$ rather than the simple formation of a sidewall oxide passivation. A relatively low oxidation annealing temperature was chosen in order to observe gradual changes in Ge NW properties that could be correlated with the change in stoichiometry of the surface oxide layer.

Figure 3(a) displays the NIR room-temperature PL spectra of the Ge NWs annealed in oxygen for different periods 

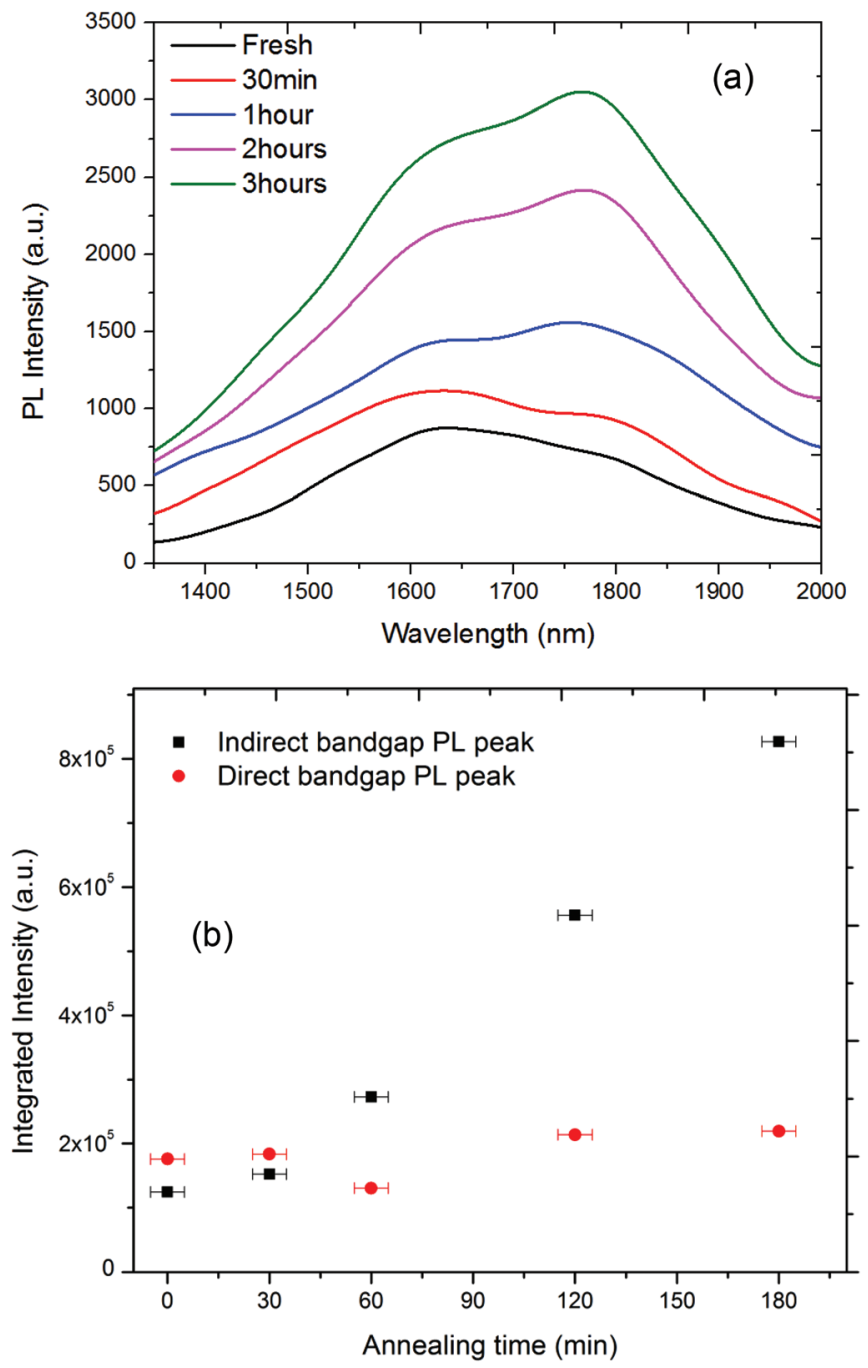

FIG. 3. (a) NIR room-temperature PL spectra of the Ge NWs, measured after different durations of furnace annealing in oxygen at $220^{\circ} \mathrm{C}$. (b) Integrated PL peak intensities of the Ge NWs as a function of annealing time in oxygen gas, measured at a nominal $300 \mathrm{~K}$.

of time including the fresh nanowire sample. The PL measurements were done at room temperature and the laser excitation power was $30 \mathrm{~mW}$. Increasing the oxidation time at $220{ }^{\circ} \mathrm{C}$ results in a clear increase in the overall band-edge photoluminescence and, in particular, increases the indirectgap feature $(\sim 1750 \mathrm{~nm})$ relative to the direct-gap feature $(\sim 1600 \mathrm{~nm})$. To explain this behavior, the PL spectra have been decomposed into two indirect bandgap transition and direct bandgap transition components whose relative contributions depend on the annealing time. As the exact shape of the PL spectra is not known, we performed decomposition into two Gaussian functions. ${ }^{33-35}$ Figure 3(b) shows the dependence of the integral under those Gaussian peaks as a function of the annealing time. It can be seen in Figure 3(b) that within the error of fitting the peaks (which is estimated to be between $1.5 \%$ and $5 \%$ ) the integral under the direct bandgap PL peak stays relatively constant while integrated intensity from the indirect bandgap PL peak increases greatly as the annealing time increases.

After $3 \mathrm{~h}$ of annealing at $220^{\circ} \mathrm{C}$, the PL spectrum is dominated by the indirect gap peak; therefore, we did not anneal the nanowires further. The increasing ratio of the
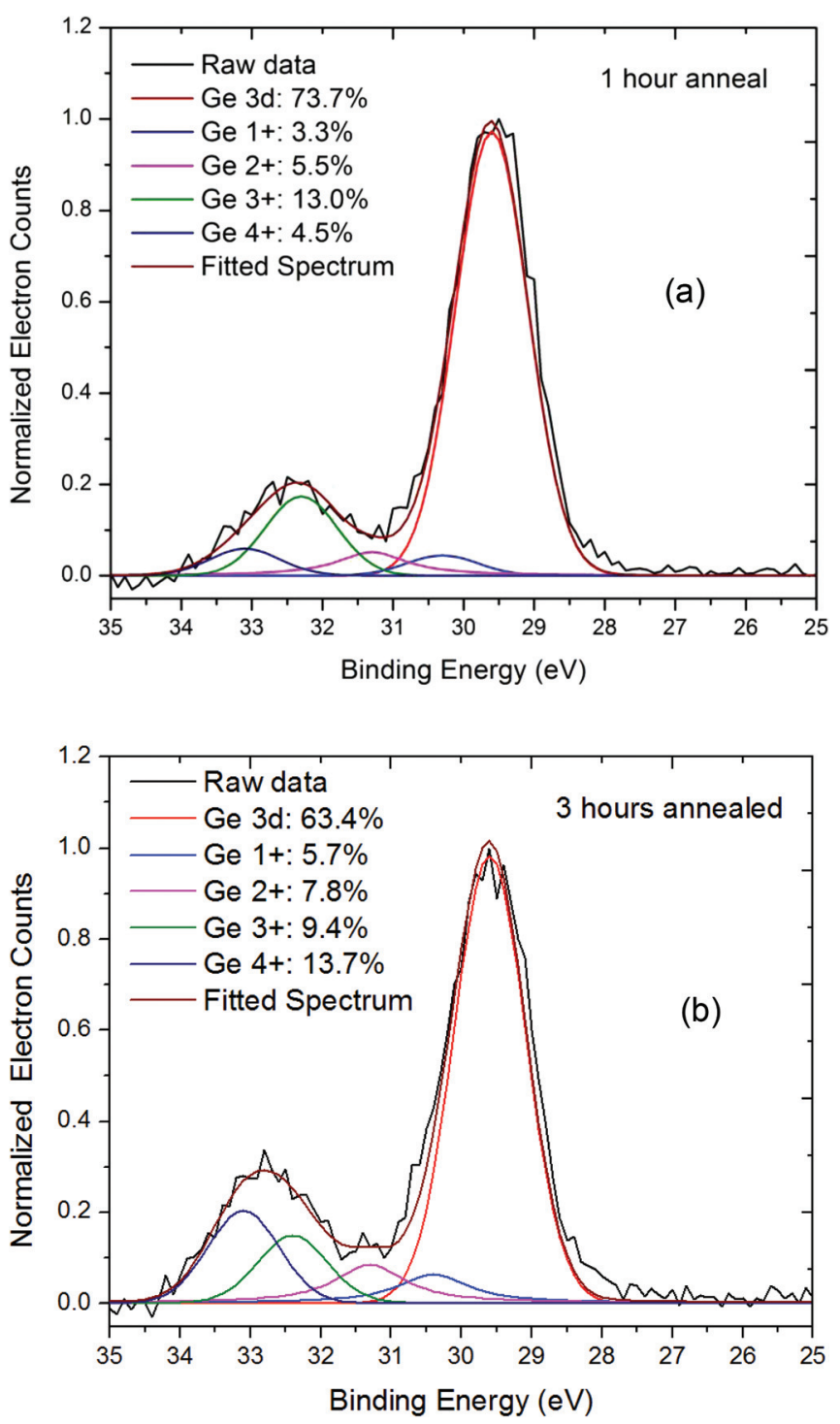

FIG. 4. Germanium oxide chemical states fitting analysis applied to the Ge $3 d$ XPS spectrum of the oxide formed by furnace annealing at $220^{\circ} \mathrm{C}$ for (a) $1 \mathrm{~h}$ and (b) $3 \mathrm{~h}$.

indirect bandgap to direct bandgap recombination peak with increasing annealing time (Figure 3(b)) suggests that this behavior may increase further until the point is reached that the oxide at the interface with $\mathrm{Ge}$ is entirely in the $\mathrm{GeO}_{2}$ form and cannot be further oxidized.

We also annealed several nanowire samples at $300{ }^{\circ} \mathrm{C}$ for up to $90 \mathrm{~min}$, and we observed a higher intensity of band edge photoluminescence PL signal as a result. No further changes in the PL spectrum were detected after annealing the NWs for longer periods at that temperature, suggesting that oxidation of the Ge NW surface during the anneal rapidly passivates fast states near the oxide/Ge interface.

We also performed the same experiments on a Si (111) wafer identical to the ones used as substrates for NW growth, with the same measurement conditions to confirm that the $\mathrm{Si}$ (111) substrate did not play any part in the observed PL from the Ge NWs. The spectrum of the Si (111) substrate shows no peaks other than one peak of wavelength consistent with the crystalline $\mathrm{Si}$ indirect bandgap: $\sim 1.11 \mu \mathrm{m}$. Therefore, the observed NIR PL in Figs. 2 and 3 originates from the Ge NWs. 
To confirm the further growth of Ge oxide on the nanowires and to determine stoichiometry changes that occur during $\mathrm{O}_{2}$ annealing of wires with an initial native oxide coating, we have performed X-ray photoelectron spectroscopy (XPS), using $\mathrm{Al}\left(\mathrm{K}_{\alpha}\right)$ radiation $(1486.7 \mathrm{eV})$ with a takeoff angle of $35^{\circ}$. The sampling depth for this measurement is estimated to be $2.9 \mathrm{~nm}$, meaning that it likely probes both the $\mathrm{GeO}_{\mathrm{x}} / \mathrm{Ge}$ interface region and the oxide and Ge regions on either side of the interface. Several studies have described the oxidation conditions for formation of the various Ge suboxide states. ${ }^{23,24}$ These results suggest that when Ge NWs are exposed to air, the native oxide of Ge will form the $\mathrm{Ge}_{2} \mathrm{O}_{3}\left(\mathrm{Ge}^{3+}\right)$ stoichiometry predominantly. This oxide state will leave the germanium surface with some dangling bonds that can trap carriers and promote their non-radiative recombination. Annealing of $\mathrm{Ge}$ in molecular oxygen is expected to promote the formation of an oxide with the $\mathrm{Ge}^{4+}$ oxidation state (identified by a chemical shift $\Delta \varepsilon=3.5 \pm 0.1 \mathrm{eV}$ with respect to the bulk contribution of the Ge $3 \mathrm{~d}$ levels ${ }^{24,40}$ ). If this oxidation reaction occurs primarily at the $\mathrm{GeO}_{\mathrm{x}} / \mathrm{Ge}$ interface, rather than at the oxide surface, a reduced interface trap density should result, ${ }^{19-21}$ leading to more radiative recombination of photoexcited carriers and stronger bandedge photoluminescence.

Figures 4(a) and 4(b) show the peak fitting analysis for the Ge $3 \mathrm{~d}$ core level peak in an XPS spectrum collected from a Ge NW assembly after $1 \mathrm{~h}$ and $3 \mathrm{~h}$ anneal in $\mathrm{O}_{2}$ gas at $220^{\circ} \mathrm{C}$, respectively. Detailed analysis of the XPS results shows that increasing the annealing time from $1 \mathrm{~h}$ to $3 \mathrm{~h}$ systematically increases the intensity of the $\mathrm{Ge}^{4+}$ component of the Ge $3 \mathrm{~d}$ peak from $4.5 \%$ to $13.7 \%$. This change is evidenced by the shift to higher binding energies observed in the Ge oxide peak, as the annealing time increases from $1 \mathrm{~h}$ (Fig. 4(a)) to $3 \mathrm{~h}$ (Fig. 4(b)).

These results confirm the increasing presence of $\mathrm{GeO}_{2}$ in the surface oxide layer as a function of the duration of the low-temperature $\mathrm{O}_{2}$ anneal. The corresponding increase in photoluminescence associated with the indirect-gap transition $(\sim 1750 \mathrm{~nm})$ suggests that the $\mathrm{GeO}_{2}$ growth occurs predominantly at the oxide/Ge interface, reducing the density of interface trap states.

The findings of these experiments are summarized schematically in Figure 5. Figure 5(a) sketches a comparison of $\mathrm{GeO}_{\mathrm{x}}$ (native oxide)/Ge and $\mathrm{GeO}_{\mathrm{x}} / \mathrm{GeO}_{2} / \mathrm{Ge}$ nanowire structures. The lower density of fast interface states in the latter results in a higher rate of radiative recombination of photoexcited carriers and consequently higher intensity of PL signal from indirect bandgap recombination. Figure 5(b) shows a band diagram of Ge with the direct ( $\Gamma$-valley) and indirect (L-valley) bandgaps and the carrier recombination process before and after the formation of $\mathrm{GeO}_{2}$ on the nanowires. After the Ge NWs are illuminated by the excitation laser beam, a majority of photogenerated electrons will populate the L-valley and a minority the $\Gamma$-valley. This minority of

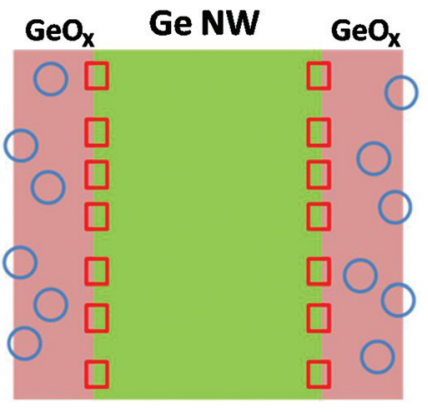

Fast surface states

Slow surface states

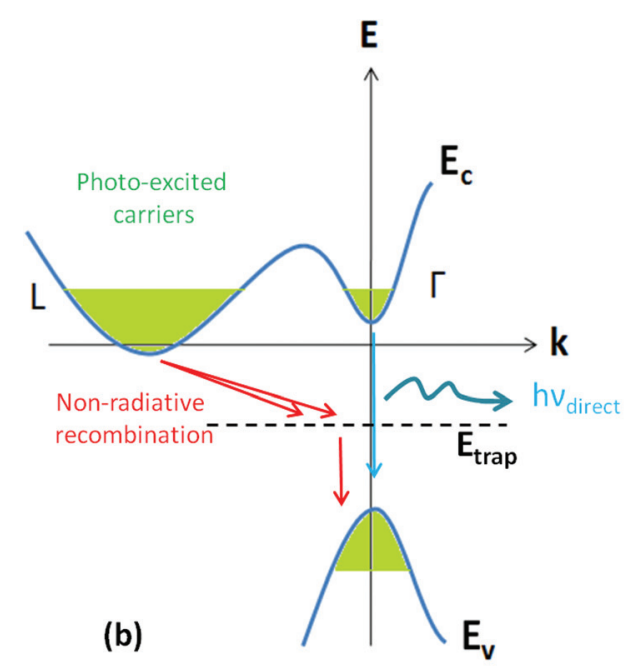

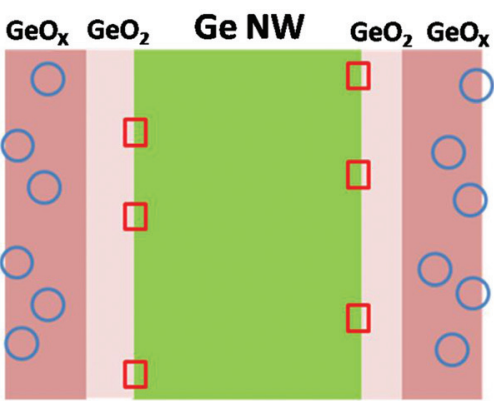

(a)

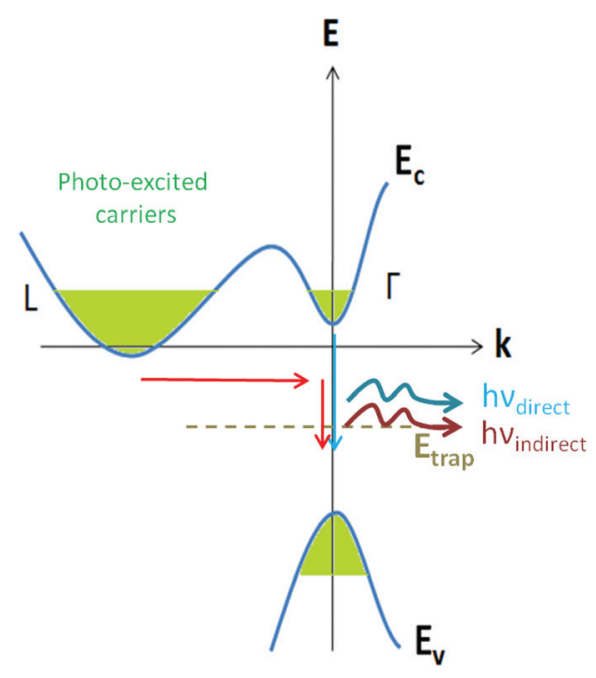

FIG. 5. (a) (Left) Ge oxide/Ge interface with both relatively fast interface traps (picoseconds-nanosecond timescale) and slower states ( $\sim$ seconds timescale) in the oxide; (right) $\mathrm{GeO}_{2} /$ Ge interface formed after oxidation annealing of the sample, with a lower density of interface states. Fig. (b) Photoexcited carriers in the Ge band diagram. (Left) non-radiative recombination of the majority of photoexcited carriers occurs via trap levels existing in the bandgap of Ge; (right) radiative recombination of both L-valley and $\Gamma$-valley electrons after the interface trap density has been reduced by the formation of $\mathrm{GeO}_{2}$. 
photoexcited carriers can recombine radiatively, producing a relatively weak detectable direct-gap PL signal that is approximately independent of the degree of surface passivation of the nanowires. ${ }^{18}$ However, the majority of excited carriers in unpassivated Ge NWs will recombine nonradiatively via interface traps. (Fig. 5(b), left). Annealing the initially native oxide-coated NWs in $\mathrm{O}_{2}$ at moderate temperatures provides an electrically more-passive $\mathrm{GeO}_{2} / \mathrm{Ge}$ interface that enhances the probability of radiative recombination of the L-valley electrons (Fig. 5(b), right).

Germanium nanowire growth and characterization were supported by National Science Foundation Grant No. DMR1206511.

${ }^{1}$ D. M. Lyons, K. M. Ryan, M. A. Morris, and J. D. Holmes, Nano Lett. 2, 811 (2002).

${ }^{2}$ S. Saito, K. Oda, T. Takahama, K. Tani, and T. Mine, Appl. Phys. Lett. 99, 241105 (2011).

${ }^{3}$ L. Colace, G. Masini, F. Galluzzi, G. Assanto, G. Capellini, L. Di Gaspare, E. Palange, and F. Evangelisti, Appl. Phys. Lett. 72, 3175 (1998).

${ }^{4}$ Y. H. Ahn and J. Park, Appl. Phys. Lett. 91, 162102 (2007).

${ }^{5}$ O. Demichel, M. Heiss, J. Bleuse, H. Mariette, and A. Fontcuberta i Morral, Appl. Phys. Lett. 97, 201907 (2010).

${ }^{6}$ J. Engvall, J. Olajos, H. G. Grimmeiss, H. Kibbel, and H. Presting, Phys. Rev. B 51, 2001 (1995).

${ }^{7}$ J. Olajos, J. Engvall, H. G. Grimmeiss, M. Gail, G. Abstreiter, H. Presting, and H. Kibbel, Phys. Rev. B 54, 1922 (1996).

${ }^{8}$ M. Bonfanti, E. Grilli, M. Guzzi, M. Virgilio, G. Grosso, D. Chrastina, G. Isella, H. von Känel, and A. Neels, Phys. Rev. B 78, 041407(R) (2008).

${ }^{9}$ Y. Chen, C. Li, Z. Zhou, H. Lai, S. Chen, W. Ding, B. Cheng, and Y. Yu, Appl. Phys. Lett. 94, 141902 (2009).

${ }^{10}$ E. Gatti, E. Grilli, M. Guzzi, D. Chrastina, G. Isella, and H. von Känel, Appl. Phys. Lett. 98, 31106 (2011).

${ }^{11}$ D. C. Paine, C. Caragianis, T. Y. Kim, Y. Shigesato, and T. Ishahara, Appl. Phys. Lett. 62, 2842 (1993).

${ }^{12}$ Y. Maeda, Phys. Rev. B 51, 1658 (1995).

${ }^{13}$ M. Zacharias and P. M. Fauchet, Appl. Phys. Lett. 71, 380 (1997).

${ }^{14}$ C. L. Yuan and P. S. Lee, Europhys. Lett. 83, 47010 (2008).

${ }^{15}$ C. L. Yuan, H. Cai, P. S. Lee, J. Guo, and J. He, J. Phys. Chem. C 113, 19863 (2009).

${ }^{16}$ K. S. Min, K. V. Shcheglov, C. M. Yang, H. A. Atwater, M. L. Brongersma, and A. Polman, Appl. Phys. Lett. 68, 2511 (1996).
${ }^{17}$ G. Audoit, É. Ní. Mhuircheartaigh, S. M. Lipson, M. A. Morris, W. J. Blau, and J. D. Holmes, J. Mater. Chem. 15, 4809 (2005).

${ }^{18}$ S. Hu, Y. Kawamura, K. C. Y. Huang, Y. Li, A. F. Marshall, K. M. Itoh, M. L. Brongersma, and P. C. McIntyre, Nano Lett. 12, 1385 (2012).

${ }^{19}$ Y. Kawamura, K. C. Y. Huang, S. V. Thombare, S. Hu, M. Gunji, T. Ishikawa, M. L. Brongersma, K. M. Itoh, and P. C. McIntyre, Phys. Rev. B 86, 035306 (2012).

${ }^{20}$ D. Riabinina, C. Durand, M. Chaker, N. Rowell, and F. Rosei, Nanotechnology 17, 2152 (2006).

${ }^{21}$ D. Kuzum, T. Krishnamohan, A. J. Pethe, A. K. Okyay, Y. Oshima, Y. Sun, J. P. McVittie, P. A. Pianetta, P. C. McIntyre, and K. C. Saraswat, IEEE Electron Device Lett. 29, 328 (2008).

${ }^{22}$ M. Houssa, G. Pourtois, M. Caymax, M. Meuris, M. M. Heyns, V. V. Afanas'ev, and A. Stesmans, Appl. Phys. Lett. 93, 161909 (2008).

${ }^{23}$ K. Kita, S. Suzuki, H. Nomura, T. Takahashi, T. Nishimura, and A. Toriumi, Jpn. J. Appl. Phys., Part 1 47, 2349 (2008).

${ }^{24}$ A. Molle, M. N. Bhuiyan, G. Tallarida, and M. Fanciulli, Appl. Phys. Lett. 89, 083504 (2006).

${ }^{25}$ H. Adhikari, P. C. McIntyre, S. Sun, P. Pianetta, and C. E. D. Chidsey, Appl. Phys. Lett. 87, 263109 (2005).

${ }^{26}$ H. Adhikari, A. F. Marshall, C. E. D. Chidsey, and P. C. McIntyre, Nano Lett. 6, 318 (2006).

${ }^{27}$ J. H. Woodruff, J. B. Ratchford, I. A. Goldthorpe, P. C. McIntyre, and C. E. D. Chidsey, Nano Lett. 7, 1637 (2007).

${ }^{28}$ P. W. Leu, H. Adhikari, M. Koto, K.-H. Kim, P. de Rouffignac, A. F. Marshall, R. G. Gordon, C. E. D. Chidsey, and P. C. McIntyre, Nanotechnology 19, 485705 (2008).

${ }^{29}$ R. H. Kingston, J. Appl. Phys. 27, 101 (1956).

${ }^{30}$ R. P. Prasankumar, S. Choi, S. A. Trugman, S. T. Picraux, and A. J. Taylor, Nano Lett. 8, 1619 (2008).

${ }^{31}$ T. Hanrath and B. A. Korgel, J. Phys. Chem. B 109, 5518 (2005).

${ }^{32}$ R. R. Lieten, K. Bustillo, T. Smets, E. Simoen, J. W. Ager III, E. E. Haller, and J.-P. Locquet, Phys. Rev. B 86, 035204 (2012).

${ }^{33}$ R. Prabakaran, R. Kesavamoorthy, S. Amirthapandianc, and F. P. Xaviera, Physica B 337, 36 (2003).

${ }^{34}$ I. L. Medintz, M. H. Stewart, S. A. Trammell, K. Susumu, J. B. Delehanty, B. C. Mei, J. S. Melinger, J. B. Blanco-Canosa, P. E. Dawson, and H. Mattoussi, Nature Mater. 9, 676 (2010).

${ }^{35}$ T. Kiba, Y. Mizushima1, M. Igarashi, S. Samukawa, and A. Murayama, Nanoscale Res. Lett. 7, 587 (2012).

${ }^{36}$ H. Y. Fan, Phys. Rev. 82, 900 (1951).

${ }^{37}$ P. B. Allen and M. Cardona, Phys. Rev. B 27, 4760 (1983).

${ }^{38}$ P. Lautenschlager, P. B. Allen, and M. Cardona, Phys. Rev. B 31, 2163 (1985).

${ }^{39}$ M. Gunji, S. V. Thombare, S. Hu, and P. C. McIntyre, Nanotechnology 23, 385603 (2012).

${ }^{40}$ D. Schmeisser, R. D. Schnell, A. Bogen, F. J. Himpsel, D. Rieger, G. Landgren, and J. F. Morar, Surf. Sci. 172, 455 (1986). 